\title{
EFEITO DE LÂMINAS DE IRRIGAÇÃO SOBRE O RENDIMENTO E QUALIDADE DA FIBRA DE CULTIVARES DE ALGODOEIRO HERBÁCEO (Gossypium hirsutum L. r. latifolium Hutch)
}

\author{
José Nunes Filhoㅁ, Vital Artur de Lima e Sá2, Ivan Souto de Oliveira Júnior ${ }^{3}$, \\ João Luiz Barbosa Coutinho ${ }^{4}$ e Venézio Felipe dos Santos ${ }^{5}$.
}

\begin{abstract}
RESUMO
Estudou-se o rendimento e a qualidade da fibra de três cultivares $(C)$ de algodoeiro herbáceo (CNPA 7H, CNPA Precoce 1 e CNPA 6H) submetidas a quatro lâminas de irrigação $\left(\mathrm{LI}_{1}=671 \mathrm{~mm}\right.$, $\mathrm{LI}_{2}=785 \mathrm{~mm} ; \mathrm{LI}_{3}=872 \mathrm{~mm}$ e $\mathrm{LI}_{4}=927 \mathrm{~mm}$ ) aplicadas em sulcos nivelados e fechados. Adotou-se o delineamento experimental de blocos ao acaso, com parcelas subdivididas, sendo a parcela principal (LI) e a subparcela (C) com quatro repetições; as equações de regressão, ajustadas aos dados de produtividade de algodão em caroço, seguiram um modelo quadrático com alta significância para a variável lâmina de irrigação, tendo-se obtido rendimentos máximos de 3051, 2763 e 2423 kg.ha-1 para as cultivares CNPA 7H, CNPA Precoce 1 e CNPA 6H, através de 836, 882 e 821mm de água total aplicada, respectivamente; já a uniformidade da fibra variou com o manejo e a intensidade de irrigação, de forma significativa, enquanto o comprimento, a resistência e a finura não foram afetados nas condições estudadas com essas cultivares.
\end{abstract}

Palavras-chave: irrigação, sulcos, produtividade, algodão

\section{EFFECT OF IRRIGATION DEPTH ON THE YIELD AND FIBER QUALITY IN COTTON CULTIVARS (Gossypium hirsutum L. r. latifolium Hutch).}

\begin{abstract}
The main objective of the trial was to evaluate the effect of irrigation depths on the yield and fiber quality in cotton cultivars. CNPA 7H, CNPA Precoce 1 , and CNPA $6 \mathrm{H}$. The irrigation depths studied were: $\mathrm{LI}_{1}=671 \mathrm{~mm}, \mathrm{LI}_{2}=785 \mathrm{~mm}, \mathrm{LI}_{3}=872 \mathrm{~mm}$ and $\mathrm{LI}_{4}=927 \mathrm{~mm}$, which were applied in closed end levelled forrows. The experimental design adopted was a completely randomized block in a split - plot layout with 4 replications. The irrigation depths were the main plots, while the cultivars were the subplots. The adjusted regression equations for cotton yield had a quadratic model, with high significance for the irrigation depths. The maximun estimated cotton yields were 3051, 2763 and $2423 \mathrm{~kg} \cdot \mathrm{ha}^{-1}$, for the cultivars CNPA 7H, CNPA Precoce 1 and CNPA 6H for a total water application of 836,882 and $821 \mathrm{~mm}$, respectively. The fiber uniformity varied significantly with the management and the intensity of irrigation. On the other hand, the length, the resistence, and the fineness were not affected by the irrigation depth.
\end{abstract}

Key words: irrigation, furrow, yield, cotton

\footnotetext{
${ }^{1}$ Eng. Agr. Dr. em Irrigação e Drenagem - Empresa Pernambucana de Pesquisa Agropecuária - IPA, Av. Gal. San Mantin 1371, Bonji - 50761-000, Recife, PE, CP 1022., E-mail:nunes @ ipa. br

${ }^{2}$ Eng. Agr. M.Sc. em Irrigação - IPA. E-mail: vital @ ipa. br

${ }^{3}$ Eng. Agr. M.Sc. em Fitotecnia - IPA. E-mail: ipa @ ipa. br.

${ }^{4}$ Eng. Agr. M.Sc. em Fitossanidade, IPA. E-mail: ipa. br @ ipa.br.

${ }^{5}$ Eng.Agr. B.Sc. em Estatística Aplicada, IPA. E-mail: ipa @ ipa.br
} 


\section{INTRODUÇÃO}

O algodão é um dos produtos básicos para a economia brasileira, em especial para a região nordestina, onde possui fundamental importância social na geração de empregos nas áreas rurais e urbanas (Beltrão et al., 1986); entretanto, como em toda região semi-árida, a instabilidade climática, representada pela irregularidade da distribuição das chuvas, faz com que uma agricultura produtiva só se possa desenvolver às custas da irrigação. Segundo Waddle (1984) mais de 60\% da área mundial com algodão são estabelecidos sob condições de irrigação. No Nordeste do Brasil, a exploração do algodoeiro herbáceo em regime de irrigação é, hoje, prioritária, devido principalmente ao estabelecimento do bicudo (Anthonomus grandis Boheman) como praga e ao atual rendimento regional, em geral baixo, o que dificulta a convivência com esta praga (Silva \& Bezerra, 1990). Os estudos de Magalhães et al. (1987) consideram de responsabilidade das irregularidades pluviométricas as perdas de até $70 \%$ na produção e produtividade do algodoeiro no semiárido.

Apesar de ser uma cultura tolerante à seca, o seu rendimento pode ser bastante reduzido quando ocorre deficiência de umidade no solo (Marani \& Amirav, 1971 e Millar, 1976).

Jackson \& Tilt (1986) constataram que, irrigando-se o algodoeiro quando já haviam sido consumidos, em média, 95, 80,65 e $50 \%$ da umidade disponível do solo, o rendimento do algodão aumentou de forma significativa com os níveis de irrigação, na ordem de 3947, 5037, 5104 e 5359 kg.ha-1, respectivamente. Resultados semelhantes foram obtidos na Paraíba, por Silva et al. (1988) quando os níveis de umidade do solo diminuíram de 75, 50 e 25\%; por outro lado, Aragão Jr. et al. (1989) trabalhando no município de Russas, CE, utilizando a cultivar BR-1, concluíram que a irrigação com base em $80 \%$ da evapotranspiração potencial e uma freqüência de 6 dias, proporcionou o maior rendimento, $1872 \mathrm{~kg} \cdot \mathrm{ha}^{-1}$, com uma lâmina média de irrigação de $752 \mathrm{~mm}$. Alves et al. (1973) revelam que a umidade do solo, elevada durante o florescimento do algodão, favorece o desenvolvimento vegetativo, em detrimento da produção e a sua escassez é prejudicial, provocando senescência. Em casos extremos, o ciclo da cultura é abreviado e a produção insignificante, mas, na fase que antecede a floração do algodão, o déficit de umidade no solo pode limitar o desenvolvimento e o rendimento da cultura (Stockton et al., 1967; Ferry et al., 1967). Por outro lado, Oliveira et al. (1992) estudando o efeito dos níveis de umidade disponível no solo, de 45, 35, 25, 15 e $5 \%$, em duas fases do ciclo da cultura do algodoeiro herbáceo, destacaram que o nível de $25 \%$ proporcionou o maior rendimento de algodão em caroço.

Cultivares de algodoeiro podem diferir na morfologia da folha, na altura e na área foliar, apresentando maior ou menor habilidade de osmorregulação, sob intenso estresse hídrico no solo. Karani et al. (1980) concluíram que as folhas do genótipo super okra tiveram sempre o menor potencial osmótico que o genótipo de folha normal, resultando num aumento do potencial de turgor, de 0,2 para 0,3 $\mathrm{MPa}$.

Nunes Filho (1993) comparando duas cultivares de algodoeiro herbáceo (CNPA Acala 1 e CNPA Precoce 1) sob diferentes níveis de umidade e salinidade do solo, concluiu que a cultivar CNPA Precoce 1 apresentou maior tendência de tolerância ao estresse hídrico e/ou salino, que a cultivar CNPA Acala 1, devido ao menor teor relativo de água na folha (TRA), ao menor potencial de água na folha e ao maior rendimento de algodão em caroço, em relação à CNPA Acala 1 , nas condições estudadas.

Considerando-se esses aspectos, o presente trabalho teve como objetivo estudar o efeito de lâminas de irrigação sobre o rendimento e características tecnológicas da fibra de cultivares de algodoeiro herbáceo (Gossypium hirsutum L. r. latifolium Hutch) na região semi-árida de Pernambuco.

\section{MATERIAL E METODOS}

O trabalho foi conduzido no período de julho a novembro de 1994, na Estação Experimental de Serra Talhada, pertencente à Empresa Pernambucana de Pesquisa Agropecuária - IPA, apresentando as seguintes coordenadas geográficas: latitude sul de $7^{\circ}$ 59' 15', longitude oeste de Greenwich de $38^{\circ} 18^{\prime}$ ' 0 " e altitude de 431m (Anuário Estatístico de Pernambuco, 1981).

O clima da região é do tipo BSW' h', de acordo com a classificação de Köppen, ou seja, muito quente, semi-árido, com estação chuvosa de verão a outono e precipitação média anual de 876mm, no período de 1958 a 1995 (Encarnação, s.d.). A irregularidade na distribuição das chuvas apresenta-se como o maior fator limitante da produção das culturas (Nunes Filho \& Sousa, 1981).

O experimento foi localizado numa encosta, com declividade média de 4\%, em solo do tipo Podzólico Vermelho-Amarelo eutrófico, cujas características podem ser observadas na Tabela 1; as cultivares utilizadas foram: CNPA $7 \mathrm{H}\left(\mathrm{C}_{1}\right)$, CNPA Precoce $1\left(\mathrm{C}_{2}\right)$ e CNPA $6 \mathrm{H}\left(\mathrm{C}_{3}\right)$. O plantio foi manual, no espaçamento de $1,00 \mathrm{~m} \times 0,20 \mathrm{~m}$, colocando-se de 6 a 8 sementes por cova, enquanto o desbaste foi realizado quando as plantas se apresentavam com altura de 15 a $20 \mathrm{~cm}$, deixando-se as duas plantas mais vigorosas por cova; as capinas foram efetuadas a enxada, de modo a se evitar a competição das ervas daninhas com a cultura nos primeiros 70 dias e os tratamentos fitossanitários, visando ao controle do pulgão e do bicudo, foram feitos com Metasystox $250 \mathrm{CE}$ e Thiodan $350 \mathrm{CE}$, respectivamente.

Tabela 1. Características físicas e químicas do solo da área experimental de Serra Talhada

\begin{tabular}{llcc}
\hline Características & Unidade & \multicolumn{2}{c}{ Valor } \\
\cline { 3 - 4 } & & $0-20 \mathrm{~cm}$ & $20-40 \mathrm{~cm}$ \\
\hline Areia grossa & $\mathrm{g} \cdot \mathrm{kg}^{-1}$ & 350 & 360 \\
Areia fina & $\mathrm{g} \cdot \mathrm{kg}^{-1}$ & 400 & 350 \\
Silte & $\mathrm{g} \cdot \mathrm{kg}^{-1}$ & 150 & 150 \\
Argila & $\mathrm{g} \cdot \mathrm{kg}^{-1}$ & 100 & 140 \\
Classificação textural & - & & Franco arenoso \\
Capacidade de campo & $\mathrm{g} \cdot \mathrm{kg}^{-1}$ & 156 & 163 \\
Ponto de murcha permanente & $\mathrm{g} \cdot \mathrm{kg}^{-1}$ & 46 & 50 \\
Densidade aparente & $\mathrm{kg} \cdot \mathrm{dm}^{-3}$ & 1,5 & 1,4 \\
pH & - & 79 & 74 \\
Ca + Mg & $\mathrm{c} \cdot \mathrm{mol}_{\mathrm{c}} \cdot \mathrm{kg}^{-1}$ & 3,9 & 2,7 \\
P & $\mathrm{mg} \cdot \mathrm{dm}^{-3}$ & 81,0 & 36,7 \\
K & $\mathrm{c} \cdot \mathrm{mol}_{\mathrm{c}} \cdot \mathrm{kg}^{-1}$ & 0,6 & 0,4 \\
Al & $\mathrm{c} \cdot \mathrm{mol}_{\mathrm{c}} \cdot \mathrm{kg}^{-1}$ & 0 & 0 \\
Matéria orgânica & $\mathrm{g} \cdot \mathrm{dm}^{-3}$ & 5,0 & 4,0 \\
CEs* & $\mathrm{dS} \cdot \mathrm{m}^{-1} \mathrm{à}^{2} 5^{\circ} \mathrm{C}$ & 1,0 & 1,6 \\
Relação de adsorção de sódio & $\mathrm{mmol}^{0} \mathrm{~L}^{0,5}$ & 3,8 & 6,2 \\
Cl & $\mathrm{mmol} \cdot \mathrm{L}^{-1}$ & 2,3 & 8,3 \\
*Condutividade elétrica no extrato saturado & & &
\end{tabular}


O delineamento experimental utilizado foi o de blocos ao acaso, com parcelas subdivididas, sendo a parcela principal (lâminas de irrigação - LI) e a subparcela (cultivares - C) com quatro repetições; os tratamentos constituíram-se de quatro níveis de irrigação $\left(\mathrm{LI}_{1}, \mathrm{LI}_{2}, \mathrm{LI}_{3} \mathrm{e} \mathrm{LI}{ }_{4}\right)$ representados por lâminas de água aplicadas conforme discriminação a seguir, além das três cultivares especificadas anteriormente, compondo um arranjo fatorial $4 \times 3$ :

LI - irrigação de 15 em 15 dias, nas fases vegetativa, floração e frutificação

$\mathrm{LI}_{2}$ - irrigação a partir do aparecimento dos primeiros botões florais até a abertura dos primeiros capulhos, sempre que o solo atingia 35\% da água disponível

$\mathrm{LI}_{3}$ - irrigação até a abertura dos primeiros capulhos, sempre que o solo atingia $35 \%$ da água disponível

$\mathrm{LI}_{4}$ - irrigação durante o ciclo da cultura, sempre que o solo atingia $35 \%$ da água disponível.

O método de irrigação utilizado foi o de sulcos fechados, em nível, com espaçamento de $1,0 \mathrm{~m}$; a água foi aplicada às parcelas por meio de mangueira com vazão conhecida e, antes do plantio, foi aplicada uma lâmina de água suficiente para elevar os primeiros $100 \mathrm{~cm}$ do solo à capacidade de campo; todas as parcelas foram irrigadas indistintamente, conforme umidade do solo coletada em pontos aleatórios em diversas parcelas, nas profundidades de $0-20 \mathrm{~cm}$ e $20-40 \mathrm{~cm}$ até o desbaste, efetuado 10 dias após a germinação; a partir desta data as irrigações foram efetuadas seguindo-se o nível de umidade do solo e/ou os tratamentos preestabelecidos e, para o controle da umidade e definição da irrigação, foi utilizado o método gravimétrico.

Procedeu-se apenas às adubações nitrogenadas duas vezes, a primeira após o desbaste e a segunda na abertura dos primeiros botões florais, com $20 \mathrm{~kg} \cdot \mathrm{ha}^{-1} \mathrm{em}$ cada aplicação.

Foram realizadas duas colheitas, a primeira quando $50 \%$ dos capulhos estavam abertos e a última em torno de 25 dias após a primeira.

Para análise de variância foram considerados os dados de rendimento das cultivares e características tecnológicas da fibra, em cada tratamento.

\section{RESULTADOS E DISCUSSÃO}

A análise de variância dos resultados de produtividade de algodão em caroço, das cultivares nas diferentes lâminas de irrigação, evidenciou que houve efeito significativo (p 0,01$)$ tanto para cultivares como para a lâmina de água aplicada. As funções de resposta das cultivares submetidas aos diferentes tratamentos (lâminas de irrigação) seguem modelos quadráticos com alta significância, conforme Figura 1. Verifica-se que a cultivar CNPA 7H superou estatisticamente a CNPA 6H e não diferiu, entretanto, da CNPA Precoce 1 no rendimento de algodão em caroço, a nível de 5\% de probabilidade, pelo teste de Tukey (Tabela 2). De acordo com as equações de regressão (Figura 1), os rendimentos máximos foram de 3051, 2763 e $2423 \mathrm{~kg} \cdot \mathrm{ha}^{-1}$ para as cultivares CNPA 7H, CNPA Precoce 1 e CNPA 6H, através de 836,882 e $821 \mathrm{~mm} \cdot \mathrm{ha}^{-1}$ de água total aplicada, respectivamente, cujas produtividades podem ser obtidas irrigando-se a cultura até a abertura dos primeiros capulhos, sempre que o solo atingir $35 \%$ da água disponível,

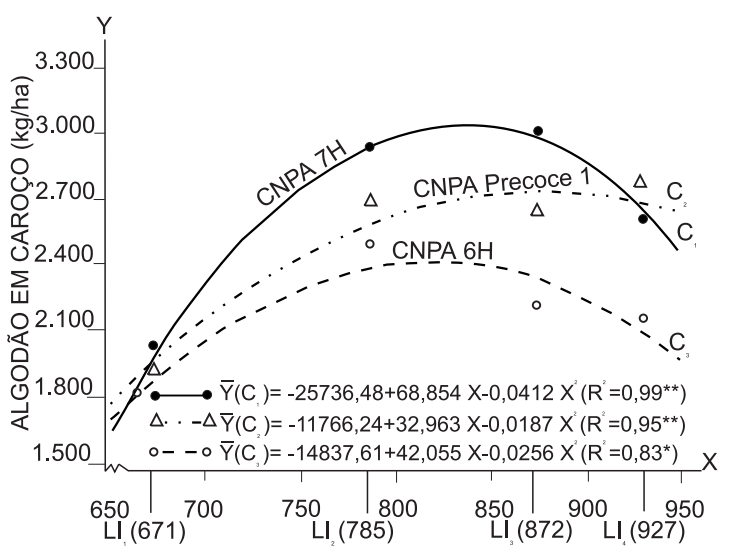

LÂMINAS DE IRRIGAÇÃO (mm)

Figura 1. Equações de regressão entre lâminas de irrigação e rendimento de algodão em caroço, de cultivares de algodoeiro herbáceo

correspondendo a um turno de rega de sete dias e a um número de 13 irrigações nesse período; resultados semelhantes foram encontrados por Oliveira et al. (1992) trabalhando com as cultivares CNPA Precoce 1 e CNPA Acala 1, num solo Aluvial franco-arenoso, do município de Sousa, PB, tendo sido realizadas 12 irrigações, também com um turno de rega de sete dias, da emergência à maturação, com rendimento médio de 3216kg.ha ${ }^{-1}$ de algodão em caroço.

Tabela 2. Rendimento de algodão em caroço (Ac), comprimento $(C)$, uniformidade $(U)$, resistência $(R)$ e finura $(F)$ da fibra, de cultivares de algodoeiro herbáceo, sob diferentes lâminas de irrigação*

\begin{tabular}{|c|c|c|c|c|c|}
\hline Fatores & $\begin{array}{l}\text { Rendimento } \\
\text { Ac (kg/ha) }\end{array}$ & $\begin{array}{l}\text { Característi } \\
\qquad \mathrm{C}(\mathrm{mm})\end{array}$ & $\begin{array}{l}\text { icas tecr } \\
\mathrm{U}(\%)\end{array}$ & $\begin{array}{l}\text { nológicas } \\
\text { R(lf/mg) }\end{array}$ & $\begin{array}{l}\text { da fibra } \\
F(m i)\end{array}$ \\
\hline \multicolumn{6}{|c|}{ I- Lâminas de Irrigação (mm) } \\
\hline $\mathrm{LI}_{1}(671)$ & $1836 \mathrm{~b}$ & $31,1 \mathrm{a}$ & $53,5 \mathrm{~b}$ & $7,5 \mathrm{a}$ & $4,1 \mathrm{a}$ \\
\hline $\mathrm{LI}_{2}(785)$ & $2445 \mathrm{a}$ & $31,3 \mathrm{a}$ & $54,5 \mathrm{a}$ & $7,3 \mathrm{a}$ & $4,3 \mathrm{a}$ \\
\hline $\mathrm{LI}_{3}(872)$ & $2405 \mathrm{a}$ & $31,6 \mathrm{a}$ & $55,0 \mathrm{a}$ & $7,3 \mathrm{a}$ & $4,3 \mathrm{a}$ \\
\hline $\mathrm{LI}_{4}(927)$ & 2466 a & $31,8 \mathrm{a}$ & $54,7 \mathrm{a}$ & $7,4 \mathrm{a}$ & $4,3 \mathrm{a}$ \\
\hline C.V. $(\%)$ & 17,5 & 2,7 & 1,1 & 4,8 & 5,5 \\
\hline \multicolumn{6}{|l|}{ II - Cultivares } \\
\hline $\mathrm{C}_{1}-\mathrm{CNPA} 7 \mathrm{H}$ & $2645 \mathrm{a}$ & $31,1 \mathrm{a}$ & $54,2 \mathrm{a}$ & $7,2 \mathrm{a}$ & $4,5 \mathrm{a}$ \\
\hline $\mathrm{C}_{2}$ - CNPA Precoce 1 & $2502 \mathrm{ab}$ & $30,0 \mathrm{~b}$ & 54,6 a & $7,0 \mathrm{a}$ & $4,0 \mathrm{~b}$ \\
\hline $\mathrm{C}_{3}-\mathrm{CNPA} 6 \mathrm{H}$ & $2182 b$ & $29,8 \mathrm{~b}$ & $54,3 \mathrm{a}$ & $7,1 \mathrm{a}$ & $4,4 \mathrm{a}$ \\
\hline C.V. $(\%)$ & 17,0 & 2,3 & 3,4 & 3,8 & 4,8 \\
\hline
\end{tabular}

*Para os tratamnetos I e II, em cada coluna as médias seguidas pela mesma letra não apresentam diferenças significativas, pelo teste de Tukey, a nível de $5 \%$ de probabilidade.

Pode-se inferir, de acordo com os resultados obtidos, que o período compreendido entre a emissão dos primeiros botões florais e a abertura dos primeiros capulhos, foi o mais sensível aos tratamentos, com maior ou menor oferta d'água, mesmo no caso do tratamento $\left(\mathrm{LI}_{2}\right)$ onde as irrigações foram efetuadas somente quando se iniciou a fase de floração; assim, a produtividade média das cultivares foi inferior em apenas $2 \%$, em relação ao rendimento máximo médio de cada genótipo estimado para as condições desse estudo, reafirmado por Millar (1976), Marinato \& Lima (1982) que citam ser o déficit de água no solo mais prejudicial durante a fase de floração do algodoeiro ou, então, o excesso de umidade durante a fase de floração e frutificação, corroborado por Marani \& Levi (1973) e Campos et al., (1992). 
Com relação às características tecnológicas da fibra, verifica-se que a análise de variância não mostrou diferenças significativas para comprimento, resistência e finura da fibra, para as diferentes lâminas de irrigação; somente a variável uniformidade da fibra foi influenciada significativamente $(\mathrm{p}<0,01)$ pela irrigação. Observa-se que no tratamento $\mathrm{LI}_{1}$, correspondente a irrigações, a cada 15 dias houve, durante todo o ciclo da cultura, redução significativa da uniformidade da fibra, em comparação com os demais tratamentos, pelo teste de Tukey, a nível de 5\% de probabilidade (Tabela 2) porém o comprimento da fibra melhorou com o aumento da umidade do solo, ocorrendo o inverso com a resistência e a finura da fibra, que diminuíram.

Resultados semelhantes foram obtidos por Bezerra et al. (1992) estudando as características tecnológicas da fibra da cultivar CNPA Precoce 1, em diferentes porcentagens de umidade disponível no solo, tendo encontrado melhor desempenho do comprimento e da finura da fibra no tratamento com reposição de água, na faixa de 25 a $50 \%$ da água disponível.

A Figura 2 mostra a curva média de resposta da uniformidade da fibra (UF) das cultivares de algodoeiro em função das lâminas de água aplicada; verifica-se que o efeito foi quadrático, tendose como máximo valor de (UF) 54,9\%, obtido com uma lâmina de $890 \mathrm{~mm}$; por outro lado, a análise de variância não detectou diferença significativa entre cultivares para uniformidade e resistência da fibra, mas houve significância $(p<0,01)$ tão somente para comprimento e finura da fibra, com superioridade da cultivar CNPA 7H sobre a CNPA Precoce 1.

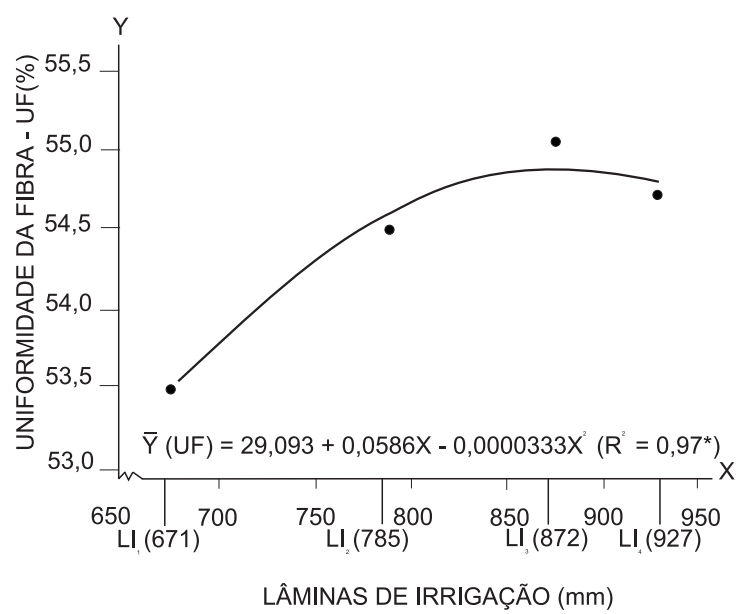

Figura 2. Efeito de lâminas de irrigação sobre a uniformidade da fibra do algodoeiro herbáceo

\section{CONCLUSÕES}

Da análise dos resultados e nas condições em que se desenvolveu o experimento, chega-se às seguintes conclusões para os genótipos testados:

1. Os rendimentos de algodão em caroço das cultivares de algodoeiro herbáceo, correlacionaram-se significativamente, de forma quadrática com as lâminas de irrigação.

2. A cultivar CNPA 7H superou estatisticamente a CNPA $6 \mathrm{H}$ em produtividade e comprimento da fibra, com rendimento máximo de $3051 \mathrm{~kg} . \mathrm{ha}^{-1}$, utilizando-se uma lâmina total de irrigação de $836 \mathrm{~mm}$, mas não diferiu da CNPA Precoce 1, em rendimento, uniformidade e resistência da fibra.
3. A uniformidade da fibra (UF em \%) dessas cultivares de algodoeiro herbáceo variou de forma significativa e quadratica com a água aplicada.

4. O comprimento e a finura da fibra aumentaram com a maior oferta de água no solo, enquanto a resistência diminuiu, embora de forma não significativa.

\section{AGRADECIMENTOS}

Os autores, em um gesto de reconhecimento à valiosa e indispensável colaboração na execução do experimento e digitação deste trabalho, estendem seus agradecimentos ao Assistente de Pesquisa Elieser Cabral da Silva, ao Assistente Rural Dilson Anacleto de Souza e às Assistentes Administrativas Maria das Graças Soares de Sousa e Ângela dos Anjos Vilela.

\section{REFERÊNCIAS BIBLIOGRÁFICAS}

ALVES, E.J.; FREIRE, E.C.; SOUZA, L.S. da. Cultura do algodão. Cruz das Almas: IPEAL, 1973. 22p. (IPEAL. Circular Técnica, 39).

ANUÁRIO ESTATÍSTICO DE PERNAMBUCO, Recife: CONDEPE, v. 30, 1981. 206p.

ARAGÃO JR.; T.C.; MAGALHÃES, C.A. de; SANTOS, C.S.V. dos. Estudo de lâminas de irrigação na cultura do algodão harbáceo. Fortaleza: EPACE 1989. 15p. (EPACE. Boletim de Pesquisa, 14).

BELTRÃO, N.E. de M.; CRISÓSTOMO, J.R.; NÓBREGA, L.B. da; SANTOS, E.O. dos; AZEVEDO, D.M.P. de; VIEIRA, D.J.; GUIMARÃES, P.M.; SILVA, M.J. da. O algodão e tecnologias disponíveis no Nordeste brasileiro. Fortaleza: Banco do Nordeste do Brasil/EMBRAPA/CNPA, 1986. 168p. (Estudos Econômicos e Sociais, 32).

BEZERRA, J.R.C.; LUZ, M.J.da S.L.; CARVALHO, O.S.; GUERRA, A.G. Efeito da adubação nitrogenada e do manejo da água sobre o algodoeiro herbáceo (Gossypium hirsutum L.). In: GONGRESSO NACIONAL DE IRRIGAÇÃO E DRENAGEM, 9., 1992, Natal, RN, Anais ... Fortaleza: Associação Brasileira de Irrigação e Drenagem, 1992, v.1, p. $1303-1318$.

CAMPOS, T.G. da S.; OLIVEIRA, F.A. de.; SANTOS, J.W. dos. Estudos da última irrigação em quatro cultivares de algodoeiro herbáceo. In: CONGRESSO NACIONAL DE IRRIGAÇÃO E DRENAGEM, 9., 1992, Natal, RN, Anais ... Fortaleza, Associação Brasileira de Irrigação e Drenagem, 1992, v. 1, p. 1689 - 1702.

ENCARNAÇÃO, C.R.F.da. Observações meteorológicas e tipos climáticos das unidades e campos experimentais da Empresa IPA. Recife: Empresa Pernambucana de Pesquisa Agropecuária, s.d. 110p.

FERRY, G.V.; GEORGE, A.G.; JONHSON, C.E.; WcCUTCHEON, O. D.; STROMBERG, L.K.; BOHER, L.J.; HOOVER, M. Guides in cotton irrigation. California: University of California, 1967. 25p.

JACKSON, L.E.B.; TILT, P.A. Effects of irrigation intensity and nitrogen level on the performance of eight varieties of upland cotton, (Gossypium hirsutum L.) Agronomy Journal, Madison, v. 60, n. 1, p. 13-17, 1986.

KARANI, E.; KRIEG, D.R.; QUISENBERRI, J.E. Water of cotton with different leaf morphology. Crop Science, Madison, v. 30, p. 421 - 426, 1980. 
MAGALHÃES, A.R.; GARAGORRY, F.L.; GASQUES, J.G.; MOLION, L.B.C.; AMORIM NETO, M.da S.A.; NOBRE, C.A.; PORTO, E.R.; REBOUÇAS, O. E. The effects of climatic variations on agriculture in Northeast to Brasil. Luxemburg-Austria, 1987. 109p.

MARANI, A.; AMIRAV, A. Effects of soil moisture stress on two varieties of upland cotton in Israel. I The coatal plain regions. Exploration Agricultural, London, v.7, n. 3, p. 213-224, 1971.

MARANI, A.; LEVI, D. Effects of moisture during early stages of development on growth and yield of cotton plants. Agronomy Journal, Madison, v.65, n. 4, p. 637-641, 1973.

MARINATO, R.; LIMA, C.A.S. Irrigação do algodoeiro. Informe Agropecuário. Belo Horizonte, v. 8, n. 92, p. 75 $81,1982$.

MILLAR, A.A. Respuesta de los cultivos al deficit de água como información basica para el manejo del riego, Petrolina: EMBRAPA - CPATSA, 1976. 62 p.

NUNES FILHO, J. Comportamento de duas cultivares de algodoeiro herbáceo (Gossypium hirsutum L. r. latifolium Hutch) em função da salinidade e umidade do solo. Botucatu: UNESP-FCA, 1993. 96p. Tese Doutorado

NUNES FILHO, J.; SOUSA, A.R. de. Efeito da subsolagam sobre a produção de sorgo granífero num solo Bruno Não Cálcico de Serra Talhada. In: ENCONTRO NACIONAL DE PESQUISA SOBRE CONSERVAÇÃO DO SOLO, 3. 1980, Recife, PE, Anais ... Recife: Universidade Federal Rural de Pernambuco, 1981. p. 238.
OLIVEIRA, F.A. de.; CAMPOS, T.G. da S.; SANTOS, J.W. dos. Variação no suprimento de água em dois períodos do ciclo do algodoeiro herbáceo. In: CONGRESSO NACIONAL DE IRRIGAÇÃO E DRENAGEM, 9., 1992, Natal, RN, Anais... Fortaleza: Associação Brasileira de Irrigação e Drenagem, 1992. v.1, p. 1443-1459.

SILVA, M.J. da; HOLANDA, F.A. de; MATIAS FILHO, J. Manejo da irrigação no algodoeiro anual no Nordeste brasileiro. In: EMBRAPA - Centro Nacional de Pesquisa de Algodão. Relatório Técnico Anual, 1985 - 1986. Campina Grande, 1988. p. 179-181.

SILVA, M.J. da; BEZERRA, J.R.C. Manejo de irrigação na cultura do algodoeiro anual no vale do Açu, RN. In: REUNIÃO NACIONAL DO ALGODÃO, 6, 1990. Campina Grande. Resumo, Campina Grande: EMBRAPA - CNPA, 1990. p. 192.

STOCKTON, J.R.; CARREKER, J.R.; HOOVER, M. Sugar,oil and fiber crops. Part IV - Irrigation of cotton and other fiber crops. In: HAGAN, K.M. Irrigation of agricultural lands. Madison: American Society of Agronomy, 1967, p.161-171. (Agronomy,11).

WADDLE, B.A. Crop growing practices. In: KOMEL, R.J.; LEWIS, C.P. Cotton. Madison: American Society of Agronomy, 1984. p.233-263 (Serie Agronomy, 24). 